\title{
Overview of Idiopathic Normal Pressure Hydrocephalus: Diagnosis and Improvement
}

\author{
Julia Chen Sun \\ University of California, San Diego, San Diego, CA, USA \\ Email: juliachensun@gmail.com
}

\begin{abstract}
Idiopathic normal pressure hydrocephalus (iNPH) is an uncommon yet potentially reversible cause of dementia in the elderly. It is characterized by cognitive deterioration, urinary incontinence, ventriculomegaly (enlarged cerebral ventricles) and gait apraxia. The diagnosis and management of iNPH requires an organized approach, starting with a comprehensive history and neurologic examination, a review of neuroimaging, and an evaluation of diagnosis. It is important to treat comorbidities in patients prior to specific iNPH testing, which includes testing patient responses to temporary CSF removal and assessing CSF hydrodynamics. In carefully selected patients, all physiological and neurological symptoms improved with a ventriculoperitoneal (VP) shunt surgery, which allows for a gradual adjustment in pressure to prevent complications caused by over-drainage.

Keywords: normal pressure hydrocephalus, ventriculoperitoneal shunt surgery, lumbar drainage, dementia, gait disturbance, $\mathrm{NPH}$
\end{abstract}

\section{Definition}

NPH is the chronic dilatation of the cerebral ventricles with a normal measured pressure of cerebrospinal fluid (CSF). Patients often develop a classic triad of symptoms, including gait impairment, urinary incontinence and dementia. While this paper focuses on the idiopathic type of NPH, it is important to note that secondary forms of this syndrome may develop due to foregoing trauma, previous subarachnoid hemorrhage, infection, mass lesion and aqueductal stenosis. ${ }^{[1-2]}$ It is estimated that $0.4 \%$ of the population over the age of 65 has been diagnosed with idiopathic NPH after developing the classic triad of neurological symptoms and enlarged ventricles in neuroimaging studies such as CT and non-invasive MRI. ${ }^{[3]}$ More definitive diagnostic testings for patient responses to CSF drainage through lumbar puncture (LP) or external lumbar drainage (ELD) have also been standardized to emulate the physiologic effect of shunt treatments. ${ }^{[1,3-4]}$

\section{Symptoms for diagnosis}

A proper diagnosis of iNPH searches for an evident, insidious onset of symptoms for at least 6 months. In the classic triad of iNPH symptoms, gait impairment is typically the presenting symptom, whereas dementia and urinary incontinence are late phenomena. ${ }^{[2]}$ Hence, iNPH diagnosis is less likely with dementia but no gait impairment. While the entire triad of symptoms does not have to be present during clinical evaluations, the prevalence of iNPH increases significantly with age, and clinical presentations vary significantly in severity and progression of symptoms. ${ }^{[5]}$

\section{Gait impairment}

In iNPH, gait impairment and balance disturbances typically occur early due to poor postural and locomotor reflexes. ${ }^{[6]}$ Patients often demonstrate difficulty with transitional movements (switching between sitting and standing) in the absence of primary sensorimotor deficits, gait initiation failure, frequent falling, unstable multi-step turns, and poor foot clearance. ${ }^{[3,5-7]}$ The standardized evaluation of gait function is typically done through a consolidation of the timed up-and-go test, Boon Scale and Tinetti score. ${ }^{[5-6]}$ Difficulties in maneuvering such as hyperreflexia due to the absence of upper motor neuron functions are atypical.

In iNPH, bladder dysfunction typically refers to urinary urgency in which patients have difficulty inhibiting the emptying of the bladder. ${ }^{[6]}$ Earlier stages of the symptom may include urinary urgency without incontinence and frequent nighttime urination, with patients fully aware of the need for urination and concerned about accidents.

\section{Dementia}

Neurologically, patients gradually develop front-subcortical dysfunctions, including daytime sleepiness, psychomotor 
slowing, apathy, daytime drowsiness, and a lack of motivation..$^{[5,7]}$ Similarly to other forms of dementia, functional losses associated with dementia in iNPH also cause patients to have difficulties in self-management, including inability to manage finances, take medications punctually, drive, and keep track of busy schedules. Additional differential diagnosis for other forms of dementia should be considered if patients exhibit symptoms such as impaired language expression, agnosia, hallucination, and failure to recognize close associates. ${ }^{[8]}$ Delirium raises concerns for medication side effects or the presence of a coexisting disorder.

\section{Neuroimaging abnormalities}

In iNPH, ventriculomegaly associated with the classic triad comprising cognitive impairment, gait disturbance and urinary incontinence can be detected by brain imaging such as computed tomography (CT) or magnetic resonance imaging (MRI). ${ }^{[1,5-8]}$ The CSF flow study by radionuclide cisternography and MRI lacks in sensitivity and specificity when diagnosing for iNPH. Despite usefulness in diagnosing neurodegenerative disorders, PET, SPECT, and other related functional neuroimaging modalities may also lack certainty in iNPH diagnosis.

The diagnosis of iNPH based on a review of neuroimaging results follows the following guidelines, usually including the first two and at least one of the rest.

(1) An Evan's ratio above 0.3. On head CT or MRI, the Evan's Ratio helps in distinguishing between hydrocephalus ex-vacuo and iNPH. ${ }^{[6]}$ The transverse diameter of the anterior horns of the lateral ventricles is compared to the internal diameter of the skull, and a ratio above 0.3 is indicative of iNPH and ventricular enlargement, but is by no means an absolute measurement. ${ }^{[6]}$

(2) Absence of macroscopic obstruction to CSF flow. Sylvian fissures and basal cisterns of the brain are usually enlarged. ${ }^{[9]}$

(3) Enlarged temporal horns of the lateral ventricles not entirely attributable to hippocampus atrophy. The narrowing of the sulci and subarachnoid spaces in the frontoparietal regions of the brain may also be observed.

(4) Callosal angle of $40^{\circ}$ and above..$^{[5,8-9]}$

(5) Evidence of altered brain water content not attributable to demyelination. Periventricular signal changes in axial MRI scans can reveal enlarged lateral ventricles with bright signals in the white matter in close proximity, which is potentially indicative of transependymal edema. ${ }^{[10]}$

\section{Work-up}

When the clinical presentation and neuroimaging findings are consistent with iNPH, patients should undergo more definitive testing for iNPH. ${ }^{[8]}$ The gold-standard diagnostic test for iNPH is either a large-volume lumbar puncture (LP), which removes 30-40 mL of CSF, or an external lumbar drainage (ELD), which is considered more accurate..$^{[3,4-8]}$ In a lumbar drain, patients have their CSF continuously removed while being observed for a minimum of three days, in contrast to the short-term observation after a transient removal of CSF with lumbar puncture. A more formal neuropsychological and physical therapy gait evaluation and a full spine MRI is also done prior to planning a LP or lumbar drain. ${ }^{[4]}$

CSF drainage is mainly rationalized for the purpose of emulating the physiologic effects of shunt surgery, since the presence of iNPH elicits a response to CSF removal and can be indicative of improvement through shunt surgery. If properly performed, lumbar drainage has a high positive predictive value: the patient's gait is most likely to respond, physicians do not have to rely on patient and family reporting, and the volume of removed CSF must be adequate enough to influence iNPH symptoms to assess improvement. However, lumbar drainage requires hospitalization in order to insert a 16-gauge spinal catheter for proper CSF drainage at a rate of $10 \mathrm{~mL} / \mathrm{h} .^{[1,7]}$

\section{Treatments}

Current treatment recommendations are based on surgical diversion of CSF. Shunts are placed into the ventricular system or the lumbar subarachnoid space to a distal site (pleural cavity or the venous system) so that the CSF can be reabsorbed. ${ }^{[1-13]}$ The most favorable option by far is the ventriculoperitoneal (VP) shunt, specifically the programmable type which allows for adjusting pressure according to symptoms and for a more gradual adjustment in pressure to prevent obstruction and other complications caused by lumbar over-drainage and subdural blood collections. ${ }^{[14]}$ Shunt valves are able to lower the pressure setting until symptom improvement and to raise the pressure setting when low-pressure complications are present. The choice of shunt valves and associated configurations should be consulted with neurosurgeons and patients.

All symptoms in iNPH are known to improve after shunt surgery, but after initial improvement, some patients may experience transient worsening of iNPH symptoms in association with other illnesses such as urinary tract infection. ${ }^{[11,13]}$ 
Insidious worsening of symptoms over weeks or months may be due to shunt malfunction or the emergence of comorbidities. While rates of acute surgical complications are low, rates of shunt dysfunction, subdural hematoma, shunt infection, intracerebral hematoma and seizure can also be relatively common. ${ }^{[13]}$

\section{References}

[1] Adams RD, Fisher CM, Hakim S, Ojemann RG, Sweet WH. Symptomatic occult hydrocephalus with "normal" cerebrospinal-fluid pressure A treatable syndrome. $N$ Engl J Med. 1965;273:117-126.

[2] Hebb AO, Cusimano MD. Idiopathic normal pressure hydrocephalus a systematic review of diagnosis and outcome. Neurosurgery. 2001;49:1166- 1186.

[3] Marmarou A, Young HF, Aygok GA, et al. Diagnosis and management of idiopathic normal-pressure hydrocephalus: a prospective study in 151 patients. J Neurosurg. 2005;102:987-997.

[4] Bradley WG. Normal pressure hydrocephalus new concepts on etiology and diagnosis. AJNR Am J Neuroradiol. 2000;21:1586- 1590.

[5] Relkin N, Marmarou A, Klinge P, Bergsneider M, Black PM. Diagnosing idiopathic normal-pressure hydrocephalus: INPH Guidelines, part II. Neurosurgery. 2005;57:S4-S16

[6] Ambarki K, Israelsson H, Wåhlin A, Birgander R, Eklund A, Malm J. Brain ventricular size in healthy elderly: comparison between Evans index and volume measurement.

[7] Thomas G, McGirt MJ, Woodworth G, et al. Baseline neuropsychological profile and cognitive response to CSF shunting for idiopathic normal pressure hydrocephalus. Dement Geriatr Cogn Disord. 2005;20:163- 168

[8] Mori E, Ishikawa M, Kato T, et al. Guidelines for management of idiopathic normal pressure hydrocephalus: second edition. Neurol Med Chir. 2012;52:775-809.

[9] Børgesen SE. Conductance to outflow of CSF in normal pressure hydrocephalus. Acta Neurochir. 1984;71:1-45.

[10] Tinetti ME. Performance-oriented assessment of mobility problems in elderly patients. J Am Geriatr Soc. 1986;34:119126.

[11] Katzen H, Ravdin LD, Assuras S, et al. Post shunt cognitive and functional improvement in idiopathic normal pressure hydrocephalus. Neurosurgery. 2011;68:416-419

[12] Kharkar S, Shuck J, Kapoor S, Batra S, Williams MA, Rigamonti D. Radionuclide shunt patency study for evaluation of suspected ventriculo-peritoneal shunt malfunction in adults with normal pressure hydrocephalus.

[13] Klinge P, Marmarou A, Bergsneider M, Relkin N, Black PM. Outcome of shunting in idiopathic normal-pressure hydrocephalus and the value of outcome assessment in shunted patients. Neurosurgery. 2005;57(3Suppl):S40-S52.

[14] Bateman GA. The pathophysiology of idiopathic normal pressure hydrocephalus Cerebral ischemia or altered venous hemodynamics? AJNR. Am J Neuroradiol. 2008;29:198-203. 